Review Article

\title{
EDIBLE SEEDS MEDICINAL VALUE, THERAPEUTIC APPLICATIONS AND FUNCTIONAL PROPERTIES-A REVIEW
}

\author{
CHANDRASEKAR R. ${ }^{1 *}$, SIVAGAMI B. ${ }^{2}$ \\ 1Department of Pharmacognosy, Seven Hills College of Pharmacy, Tirupati, Chittoor, Andhrapradesh, ${ }^{2}$ Department of Pharmaceutical \\ Analysis, Seven Hills College of Pharmacy, Tirupati, Chittoor, Andhrapradesh \\ "Email: sivagamib_27@rediffmail.com
}

Received: 14 Mar 2021, Revised and Accepted: 11 May 2021

\begin{abstract}
India has a rich source of tropical fruits containing edible seeds such as chia, hemp, sesame, pumkin, sunflower, mustard, nigella, guava, papaya, mangosteen, honeydew, pomegranate, fennel, fenugreek, cumin, sweet orange, cucumber, jackfruit, mango, melons, avocado and many more. These products such as the seed kernel, which constitutes about $10-35 \%$ of the weight, offer high nutritional value and therapeutic applications. This article explores the nutritional, medicinal, therapeutic applications, functional properties and bioactive constituents of the seeds of some fruits, which are analyzed for their functions and applications as sources of food value and bioactive phytochemical constituents. The seeds contain essential bioactive components such as alkaloids, carotenoids, flavonoids, glycosides, saponins, terpenoids, tannins, steroids and polyphenolic compounds and that exhibit excellent anti-inflammatory, antioxidant properties, anticancer, anti-diabetic, anti-hyperlipidemic, anti-obesity, neurological disorders, cardiovascular, skin diseases and chronic diseases. They have remarkable physicochemical properties and a high content of carbohydrates, fats, proteins, vitamins, and minerals. However extensive research activities can be carried out to determine the efficacy of the nutritional and bioactive components in different seed types, the bioavailability and potency. Extensive research with the seed parts can be investigated to identify the medicinal and functional potentials of these fruit seeds. This review gives an overview on the therapeutic applications and functional properties of seeds present in fruits, vegetables and medicinal plants. The medicinal and nutritional value, phytochemical composition, bioactive phytoconstituents, therapeutic activity, therapeutic applications and uses, proximate analysis, functional properties,
\end{abstract} analytical methods, spectroscopic methods and human clinical trials of some edible seeds are discussed in this review.

Keywords: Edible seeds, Bioactive phytoconstituents, Nutritional value, Therapeutic applications, Phytochemical composition

(C) 2021 The Authors. Published by Innovare Academic Sciences Pvt Ltd. This is an open access article under the CC BY license (https://creativecommons.org/licenses/by/4.0/) DOI: https://dx.doi.org/10.22159/ijpps.2021v13i7.41436. Journal homepage: https://innovareacademics.in/journals/index.php/ijpps.

\section{INTRODUCTION}

India is one of the largest producers and exporter of fruits in the world. India is well-known for its cultivation of rich fruit varieties with over many different species distributed throughout the country. Different varieties of major and minor fruits species and tropical fruits are cultivated and grown in the country. However, there are seeds present in fruits which include chia, hemp, sesame, pumkin, sunflower, mustard, nigella, guava, papaya, mangosteen, honeydew, pomegranate, fennel, fenugreek, cumin, sweet orange, cucumber, jackfruit, mango, melons, avocado and many more which are better known for large scale commercial cultivation. Fruit seeds are usually consumed by humans and thrown out as waste for processing. Processing of fruit usually generates a large amount of seeds as wastes. They are generally well-known for their nutritional and medicinal values, which include vitamins, proteins and carbohydrates. These fruits seeds are also processed into different by-products which has food value and food flavors. Studies were undertaken to explore their potential uses due to their high nutritional value and food applications. Many fruit seeds have been evaluated for their use in food industries and newer seed resources for the production of oils. [1]

The oil present in seeds is one of the most important components necessary for maintaining human health and diet since they are obtained in the form of renewable energy resource. Seed oils can be obtained by different extraction techniques such as hydraulic press, cold press, solvent extraction and refining. There is a high demand for seed oils and much interest is being focused on the possibilities of exploring newer seed resources for the production of oils. The quality characteristics of oils from different sources depend mainly on their fatty acid and triglyceride compositions. Apart from their nutritional status in human health, seed oils have food value, used as flavoring agents, excipients, additives in dosage forms and formulations in the preparation of topical formulations. Since there has been an increasing demand and nutritional value for healthier oils with higher proportion of saturated and unsaturated fatty acids. [2] The article aims to present useful information on the utilization of various fruit seeds as raw materials for the preparation of food processing industries. The review article was compiled using various search criteria which include Pubmed, Scopus, Science Direct, Google scholar, etc. The articles related to the review were compiled from the last $10 \mathrm{y}$ from 2010 to 2020 .

Pharmaceutical importance of few important seeds are presented in this article

Chia seeds are the edible seeds of a flowering plant from the mint family. The seeds are used as anti-diabetic, antihypertensive, heart disease and skin diseases. Hemp seeds are a rich source of proteins. Hemp is related to cannabis plant, hemp seeds have a compound called as Tetrahydrocanabinol. The seeds have been used for topical formulations to treat certain skin diseases. They contain essential fatty acids and other nutritional requirements. Hemp seeds are used for treating rheumatoid arthritis, constipation, atherosclerosis, eczema and other chronic diseases. Sesame seed is used to treat hypertension, diabetes, high cholesterol, heart disease and many other chronic conditions. Pumkin seeds are rich in unsaturated fatty acids, protein, vitamins and minerals that reduce risk factors of chronic diseases and cancer. Pumpkin seeds are rich in antioxidants, anti-inflammatory effects. Pumpkin seeds lower the risk of diabetes, anti-cancer properties, and can stop the growth of cancer cells. Sunflower seeds are obtained from the flower heads of the sunflower plant. The seeds are covered with a black and white shell they are white in color and have a soft and smooth texture. They are known for their flavoring and high nutritional value, and the seeds can be consumed raw, roasted, or incorporated into other food items. Sunflower seeds have anti-inflammatory properties. Sunflower seeds contain flavonoids, vitamins and other plant compounds that 
can fight many diseases. Sunflower seeds are rich in fatty acids, including polyunsaturated fatty acids and monounsaturated fatty acids. Consumption of sunflower seeds lowered the rates of cardiovascular disease, lowers bad cholesterol and hypertension. Sunflower seeds are rich in vitamins and minerals that can boost our immune system to fight against viruses.

\section{Hepatic and intestinal function of Chia seeds}

Chia seeds Salvia hispanica belonging to Lamiaceae family contains linoleic acid and $\alpha$-linolenic acid which increased bone mineral content and improved hepatic and intestinal morphology in sprague-dawley rats [3].

\section{Estimation of hemp seed oil by GC-MS and LC-MS methods}

Hemp seed Canabis sativa belonging to Cannabaceae family contains linoleic acid (la) and-linolenic acid (lna), caryophyllene, myrcene,sitosterol and trace amounts of methyl salicylate and cannabidiol (cbd) were detected. Hemp seed oil was analyzed by GC-MS and LCMS and Bioassays were performed with the oil to determine its effectiveness as an antimicrobial agent [4].

\section{Estimation of hemp seeds by LC-MS method}

Canabis sativa seeds contain tetrahydrocannabinol and cannabidiol, and other 30 cannabinoids were identified for the first time in hemp seed oil. Cannabinoid profile was estimated by a liquid chromatography method coupled to high-resolution mass spectrometry LC-MS [5].

\section{Antioxidant and antiproliferative activity of sesame seeds}

Sesame seeds Sesamum indicum belonging to pedaliaceae family contains phenolics and lignans. The antioxidant and antiproliferative activities were determined against HepG2 cells. The nonlignan components in bound phenolics contributed to the antioxidant and antiproliferative activities [6].

\section{Antioxidant activity of Sesame seeds}

Sesamum indicum seeds were extracted with methanol, ethanol or acetone and the extracts contain phenolics and flavonoids. The antioxidant, phytochemical and physicochemical properties were determined. Sesame seeds possess significant antioxidant activity [7].

\section{Antioxidant and antibacterial activity of sesame seeds}

Antioxidant, free radical scavenging and antibacterial properties were determined in sesame seeds Sesamum indicum seed oil sesamin, sesamolin and lignans were present in sesame seed oil. Sesamol is the best antioxidant and free radical scavenger amongst the molecules studied. Antibacterial assays against food-borne pathogens revealed sesamol to be an antimicrobial agent [8].

\section{Antioxidant activity of sesame seeds}

Sesamum indicum contains phenolic acids (hydroxybenzoic acids and hydroxycinnamic acids), flavonoids, and lignans. The antioxidant activity was estimated by reversed-phase highperformance liquid chromatography coupled to diode array detector and quadrupole-time-of-flight-mass spectrometry RP-HPLC (DAD) Q-TOF MS. Organic acids and some nitrogenous compounds also were characterized. The total phenol content and the antioxidant activity of the extract were determined [9].

The antioxidant activities of two varieties of sesame seeds were determined in Sesamum indicum seeds contains phenolics as well as flavonoids. The antioxidant potential was determined using DPPH, ABTS, and FRAP assays, total phenolic content (TPC) and total flavonoid content (TFC) were determined by ultraviolet (UV)-Vis spectrophotometry and analyzed by Pearson's method. Phenolic compounds in black sesame seeds were found to be the major contributors to antioxidant activities by using ABTS and FRAP methods [10].

Sesamum indicum seeds containing amino acids, total phenolics, sesamine, sesamolin, catechin, epicatechin and sinapic acids were identified and antioxidant potential was determined. Amino acids also remarkably increased in sprouted white and black seeds, arginine and tryptophan exhibited the greatest variations [11].

\section{Comparative study of two varieties of pumpkin seeds}

Pumkin seeds Cucurbita maxima belonging to Cucurbitaceae family containing fatty acids and amino acids were identified by GC/MS and amino acid analyzer. Comparative study of nutrient contents was determined in different parts of two varieties of pumpkin. The saturated fatty acid such as capric acid, myristic acid, and stearic acid, unsaturated fatty acids oleic, linoleic and linolenic acid were present. A significant amount of threonine, serine, methionine, isoleucine and tyrosine were present in the seed but only alanine in the seed of hybrid variety [12]

\section{Wound healing Activity of pumkin seeds}

Wound healing property was studied in Cucurbita pepo L. seeds containing tocopherols, fatty acids, and phytosterols. The results obtained exhibited excellent wound healing activity [13].

\section{Atherosclerotic activity of pumkin seeds}

Atherogenic diet-induced atherosclerosis was investigated in Cucurbita pepo L. seeds containing L-arginine, the total cholesterol TC, Low Density Lipoprotein-C, High-Density Lipoprotein-C, serum lipid concentrations in atherogenic rats was determined. Atherogenic rats supplemented with pumpkin seeds showed a significant decrease in their serum concentrations of total cholesterol and Low-Density Lipoprotein LDL-C. Pumpkin seeds supplementation has a protective effect against atherogenic rats [14].

\section{Anthelmintic efficiency of pumkin seeds}

In vitro and in vivo anthelmintic efficiency of aqueous and ethanol extracts on Cucurbita pepo L. seeds was determined in containing cucurbitine, aminoacids, fatty acids, and-for the first time-berberine and palmatine were identified. Raman, IR and LC-MS spectroscopy analyses were performed. Pumpkin seed extracts may be used to control of Gastrointestinal (G. I.) nematode infections [15].

\section{Anticancer activity of pumkin seeds}

The anticancer activity was determined in Cucurbita pepo L. belonging to Cucurbitaceae Pumpkin seed extract containing 1, 2dimethylhydrazine $(\mathrm{dmh})$ induced colon cancer in wistar rats. Pumpkin seed may prevent the risk of CC when consumed in dietary proportions [16]

\section{Chemical and nutritional characterization of pumkin seeds}

In this study, the general chemical compositions and some bioactive components, such as tocopherols, carotenoids, and $\beta$-sitosterol, were analyzed in three major species of pumpkin Cucurbita maxima L. containing monounsaturated fatty acids and polyunsaturated fatty acids, triacylglycerol, palmitic, stearic, oleic and linoleic acids [17].

\section{Anti-obesity activity of sunflower seeds}

Anti-obesity activity was determined in sunflower seeds Helianthus annuus belonging to Asteraceae family containing chlorogenic acids. Consumption of sunflower extract has a beneficial effect on body weight, fat mass, and lipid profile [18].

\section{Antioxidant activity of sunflower seeds}

Antioxidant activity and phenolic profile were investigated in Helianthus annuus containing mono-and dicaffeoylquinic acid isomers and caffeic acid hexose, were identified using the LC-TOFMS/MS technique [19].

Black mustard is used for common cold, rheumatoid arthritis, gout, respiratory disorders and other conditions. Black mustard seed has been used for asthma, arthritis, headache, nasal congestion, intestinal worms and toothache. Watermelon seeds Citrullus lanatus part of the plant family Cucurbitaceae, watermelon is largely cultivated in many parts of the world. There are currently more than 1,000 varieties available. Watermelon is rich in antioxidants and vitamins. It contains the compound lycopene, which is important for heart and 
may protect the skin from UV rays. Watermelon contains citrulline a compound which has excellent source of amino acid. The citrulline found in watermelon increase immunity to protect the body against viruses. Citrulline deficiency may lead to decrease immune response and also improves cardiovascular risk factors. Musk Melon is a semisweet fruit that belongs to the watermelon and honeydew melon. It also belongs to the same plant family as cucumbers, pumpkins etc. The antioxidant present in musk melon has anti-inflammatory property. They have free radicals scavenging activity that cause oxidative stress and cell damage, reducing risk of developing serious health issues such as diabetes, heart disease, arthritis and more. Musk melon contains two vitamins $\mathrm{A}$ and $\mathrm{C}$ both of these vitamins play a major role in maintaining healthy skin. Vitamin A helps to protect the skin from UV radiations and vitamin C helps to support the production of natural collagen. Musk Melon has two carotenoid compounds lutein and zeaxanthin, two compounds that give fruits and vegetables their yellow and red colors. Combined with vitamin A, these antioxidants play an important role in protecting vision and eye health. Beta carotenes, found in Musk melon as vitamin A, may help prevent respiratory problems such as bronchial asthma. Choline, an antioxidant found in Musk melon, may also help to reduce inflammation in people with chronic bronchitis.

Pomegranate can help protect heart diseases and may even prevent cancer. Pomegranate seed is used for the treatment of hypertension, energy booster, heart disease, diabetes, and many other conditions. Fennel seed is a spice harvested from the herb fennel plant. They have a sweet flavor, fennel seeds have traditionally been used as a spice in cooking. However, they can be used in all types of food, uses of fennel seeds in recipes. Fennel seeds are taken orally in the form of spice for indigestion, constipation, lowers cholesterol, menstrual cramps and symptoms of menopause.

\section{Anticancer activity of mustard seeds}

Mustard seeds Brassica nigra belonging to Brassicaceae family in which sinigrin and myrosinase were present, the anticancer activity was determined by orthotopic rat bladder cancer model [20].

\section{Antioxidant activity of mustard seeds}

Antioxidant activity of Brassica Juncea was determined containing ascorbate, riboflavin, and polyphenols, gallic acid, caffeic acid, quercetin and kaempferol dry seeds, and they were identified by HPLC analysis of the extract [21].

Antiproliferative, proapoptotic, antioxidant and antimicrobial effects of mustard seeds

The antiproliferative, proapoptotic, antioxidant and antimicrobial effects were determined in Sinapis nigra L. and Sinapis alba L. Glucosinolate, sinalbin and sinigrin were identified, Antiproliferative activity was correlated to Mitogen-Activated Protein Kinases modulation, which was cell and extract-dependent. Cell-cycle analysis evidenced a proapoptotic effect of $s$. alba on both tumor cell lines and of s. nigra only on HCT 116 cell lines [22].

\section{Antioxidant capacity of mustard seed}

Antioxidant capacity was investigated in Brassica nigra and Brassica alba. The fatty acids oleic, linoleic and linolenic were identified, whereas for yellow mustard seed the major fatty acid were erucic $(6.87 \%)$, oleic $(5.08 \%)$ and linoleic $(1.87 \%)$ acids [23].

\section{Anticancer effects of mustard seed}

Anticancer effects of Brassica nigra ethanolic extract were determined on A549 and h1299 human non-small cell lung cancer cell lines. B. nigra extract showed a substantial growth-inhibitory effect as it reduced the viability and clonogenic survival of A549 and H1299 cells in a concentration-dependent manner. B. nigra seed extract may be an important anticancer potential against human lung cancer [24].

\section{Antiasthmatic activity of mustard seed}

A pilot study was performed on respiratory tract infections, Brassica nigra mustard seed footbaths were used for determining respiratory tract infections. 103 patients were included in the intervention group and 36 patients were included in the control group. Participants of the intervention group who used mustard seed footbaths for six consecutive days showed an improvement in four of the five subscales of the Herdecke Warmth Perception Questionnaire HeWEF questionnaire [25].

\section{Antihyperlipidemic activity of nigella seeds}

Lipid-lowering effects were studied on Nigella seeds, Nigella sativa lowered total cholesterol triglyceride, low-density lipoprotein, increased high-density lipoprotein. The present study demonstrates that 8-week aerobic training plus $N$. sativa supplementation has a synergistic effect in improving lipid profile parameters [26].

\section{Fatty acid profile of watermelon and muskmelon seeds}

Watermelon and muskmelon Citrullus lanatus belonging to cucurbitaceae family containing saturated fatty acids and unsaturated fatty acids tetrdecanoic acid, pentadecanoic acid, hexadecanoic acid, heptadecanoic acid and octadecanoic acid were determined by Gas chromatography-mass spectroscopy [27].

\section{Antioxidant activity of watermelon seeds}

Citrullus lanatus containing Linoleic, stearic, palmitic and oleic acid myristic, heptadecanoic, arachidic, 9-hexadecenoic and 14eicosenoic acid. Total phenolics, antioxidant activity, peroxide value and oxidizability effects were determined [28].

\section{Hypercholesterolemia-induced atherosclerosis of watermelon seeds}

Hypercholesterolemia-induced atherosclerosis in mice was determined in Citrullus lanatus containing citrulline. C. lanatus 'sentinel' extract led to reduced body weight gain, decreased plasma cholesterol concentrations, improved homeostasis of pro-and anti-inflammatory cytokines, and attenuated development of atherosclerosis without affecting systolic blood pressure in hypercholesterolemic mice [29].

\section{Tramadol-induced testicular damage of pomegranate seed}

Tramadol-induced testicular damage in adult and adolescent rats was investigated on pomegranate seed extract. This effect may be due to the high amount of antioxidant compounds present in the pomegranate seeds [30].

\section{Effects of pomegranate seed on human epidermal keratinocyte}

Effects on human epidermal keratinocyte and human dermal fibroblast functions were determined in pomegranate seed oil Punica granatum. These results suggest heuristic potential of pomegranate fractions for facilitating skin repair in a polar manner, namely aqueous extracts promoting regeneration of dermis, and pomegranate seed oil promoting regeneration of epidermis [31].

\section{A double-blind clinical trial of pomegranate seed}

A randomized, double-blinded, placebo-controlled, balanced, crossover trial was performed on pomegranate (punica granatum) extract. PE may be effective in improving performance outcomes at maximal effort and might help to restore force in the damaged muscles [32].

\section{Ovarian-ischemia and reperfusion injury of pomegranate seed}

Ovarian-ischemia and reperfusion injury in rats was studied in pomegranate seed oil Punica granatum. While NADPH oxidase activity, MDA and TNF- $\alpha$ levels were significantly increased, SOD activity and GSH levels were reduced in ischemia and I/R groups [33].

\section{Anti-inflammatory effects of fennel seed}

Anti-inflammatory effects of fennel in the model of lipopolysaccharide (lps)-induced acute lung injury was determined. Fennel effectively blocked the inflammatory processes induced by LPS, by regulating pro-inflammatory cytokine production, transcription factors, and NO [34].

\section{Anti-obesity activity of fennel seed}

Anti-obesity placebo-controlled, single-blinded, randomized, and 3way crossover design was performed on Foeniculum vulgare Fennel tea (ft), fenugreek tea (fgt). Subjective appetite, hunger, fullness, 
desire to eat, and prospective food consumption were measured at seven independent time point using a visual analog scale (VAS). Drinking the FT and FGT were significantly effective aid to suppress subjective appetite among overweight women [35].

\section{A double-blind clinical trial of fennel seed}

A double-blind, randomized, placebo-controlled trial was performed using Foeniculum vulgare fennel on body composition in menopausal women. Bodyweight, body mass index (BMI) as well as fat distribution was measured. Menopausal women in fennel group experienced a slight increase in body weight and fat distribution [36].

\section{Antioxidant and anticancer activity of fennel seed}

Foeniculum vulgare methanolic extract contains flavonoids, terpenoids, alkaloids, phenols, and sterols gallic acid, the phenolic compound l-limonene was the most prevalent monoterpene hydrocarbon. Antioxidant, cytotoxic, and antitumor activity in breast cancer cell line (mcf7) and liver cancer cell line (hepg-2) were determined [37].

\section{Polycystic ovary syndrome of fennel seed}

Polycystic ovary syndrome (PCOS) treatment and renoprotective effect in aqueous extract of Foeniculum vulgare were determined. The mean values of blood urea nitrogen in PCOS rats treated with low dose of aqueous extract of fennel and estradiol valerate (EV) and nontreated, was significantly increased compared with non-PCOS and PCOS rats treated with high dose of aqueous extract fennel (AEF) [38].

Fenugreek seed is taken orally for diabetes, menstrual problems, high cholesterol and many other conditions. Guava seed is a tropical fruit that grows in dry or humid conditions. Guava seeds are used for intestinal cramps, spasms, antispasmodic, pain, diabetes and wound healing. The fruit is used for high blood pressure. Papaya seed is taken by mouth for cancer, diabetes, a viral infection called human papilloma virus (HPV), dengue fever and other conditions. Honey dew contains Vitamin C, high fiber content and other nutrients. Honeydew seeds help in strengthening bones and treats osteoporosis. Honeydew contains many nutrients that are essential for bone health, including folate and Vitamin K. Folate helps to break down homocysteine a compound in the body, high levels of homocysteine which can weaken bone mineral density. Vitamin $\mathrm{K}$ helps the protein called osteocalcin found in bones to bond with calcium ions. Both the folate and Vitamin $\mathrm{K}$ found in honeydew can help strengthen bones while preventing them from losing bone density. Apart from Vitamin C, it also contains high water content it is an excellent source for maintaining healthy skin. Mangosteen seeds are used for obesity and gum infection such as periodontitis. It is also used for strengthening muscles, diarrhea and several skin diseases.

\section{Hyperlipidemic activity of fenugreek seeds}

Hyperlipidemia effects of high fat diet on gut microbiota in mice were studied in fenugreek seeds Trigonella foenumgraecum. Fenugreek attenuated HFD-induced hyperlipidemia and stabilized glucose tolerance without affecting body weight. Fenugreek specifically reversed the effects of HFD and metabolic function [39].

\section{Antidiabetic activity of fenugreek seeds}

Fasting blood glucose levels and glycosylated hemoglobin (hba1c) levels in patients of type 2 diabetes mellitus controlled with diet was determined in Trigonella foenum-graecum. Fenugreek seeds have a synergistic effect on diet control exercise and fasting blood glucose and HbA1c [40]

\section{Anti-inflammatory and anti-arthritic activity of fenugreek seeds}

Anti-inflammatory and anti-arthritic activities were performed on Trigonella foenum-graecum petroleum ether extract of fenugreek seeds containing linolenic and linoleic acids. Fenugreek seeds have significant anti-inflammatory and anti-arthritic activities which are due to the presence of linolenic and linoleic acids [41].

Antioxidant activity of guava seeds
Guava seeds Psidium guajavabelonging to Myrtaceae family containing chlorogenic acid phytosterols stigmasterol, $\beta$-sitosterol and campesterol Lipid, phytosterol and phenolic compositions. The antioxidant activity, and the hepato-and neuroprotection of hydrogen peroxide-induced oxidative stress levels in the gso extract were determined [42].

\section{Antioxidant activities of papaya seeds}

Antioxidant activities of the $c$. papaya seeds water extract against hydrogen peroxide $\left(\mathrm{h}_{2} \mathrm{O}_{2}\right)$ oxidative stress in human skin detroit 550 fibroblasts was determined in Papaya seeds Carica papaya belonging to Caricaceae family [43].

\section{Antihelminthic and anti-amoebic activity of papaya seeds}

Antihelminthic and anti-amoebic activities against human intestinal parasitosis were investigated in Carica papaya Elixir composed with air-dried c. papaya seeds and honey. Thus, air-dried C. papaya seeds are efficacious in treating human intestinal parasites and without significant side effects [44].

\section{Antioxidant activities of papaya seeds}

Antioxidant activities of ethanol, petroleum ether, ethyl acetate, $n$ butanol and water extract were determined in Carica papaya seed, containing $p$-hydroxybenzoic acid (1) and vanillic acid (2), total phenolics and total flavonoids [45].

\section{Antibacterial effect of papaya seeds}

Antibacterial effect was evaluated on methanol extract of Carica papaya papaya seed. The growth or inhibition of control strains of Escherichia coli, Staphylococcus aureus, Salmonella typhi, and Pseudomonas aeruginosa as well as the clinical isolates of these bacteria were determined in growth media [46].

\section{Antioxidant activity of honey dew seeds}

Honey dew Cucumis melo belonging to Cucurbitaceae family, five phenolic compounds in water extract namely gallic, hydroxybenzoic acid and catechin and caffeic acid, nine phenolic compounds identified in methanol-water extract two vanillic acid derivatives, ellagitanins, quercetin-3-rutinoside, derivatives of syringic acid and ellagic acid. Antioxidant activity was determined, phenolic compounds were identified using a reversed-phase high performance liquid chromatography with diode array detection (HPLC-DAD) method [47].

\section{Total phenolic content and antioxidative activity of honeydew} seeds

Total phenolic content and antioxidative activities were determined in Cucumis melo containing phenolic and flavonoid compounds 3hydroxybenzoic acid, chlorogenic acid, neochlorogenic acid, isovanillic acid, apigenin-7- $\alpha$-glucoside, luteolin-7-o-glucoside, and quercetin-3-galactoside were analyzed using HPLC [48].

\section{Antioxidant activity of honey dew seeds}

Antioxidant activity was determined in Cucumis melo evaluating total polyphenols, ortho-diphenols, flavonoids, and tannins content [49].

\section{Anticancer activity of honeydew seeds}

High-performance liquid chromatography (HPLC) was used to examine and assess the contents of phenolic acid (gallic acid) and flavonoid (rutin) compounds in Cucumis melo. GC-MS analysis 3(4,5-dimethylthiazol-2-yl)-2,5-diphenyl tetrazolium bromide (mtt) was used to assess the cytotoxicity effect against pc3, hct116, hela, and jurkat cell lines [50].

\section{Human metabolic syndrome of mangosteen seeds}

Mangosteen seeds Garcinia dulcis belonging to Clusiaceae family containing garcinol, morelloflavone and citric acid were assessed for rat model of human metabolic syndrome [51].

Antioxidant and anti-inflammatory effects of mangosteen seeds 
A randomized, double-blind, placebo-controlled clinical trial was performed on Garcinia mangostana and antioxidant and antiinflammatory and immunity biomarkers in plasma of healthy adults were determined. The mangosteen significantly increased antioxidant capacity and possesses anti-inflammatory benefits with no side effects on immune, hepatic and renal functions [52].

\section{GC-MS estimation of mangosteen seeds}

Garcinia mangostana containing anthocyanins, saturated palmitic acid, stearic acid, unsaturated fatty acids, oleic acid, linoleic acid were analyzed using Gas chromatography-flame ionized detector [53].

Mango seed is used as anti-diabetic, anti-obesity, reduces high cholesterol, etc. The antioxidants, minerals and vitamins present in mango can provide important health benefits to human beings. Vitamin K helps prevent anemia and blood clot. It also plays an important role in the strengthening of bones. Mangos are also rich in vitamin C, which is important for forming healthy collagen. Mangos can also provide other health benefits like, anticancer activity lower risk of cancer, mangos contain beta-carotene, a pigment responsible for the color of the fruit. Mangos are also helpful for supporting cardiovascular system. They are a great source of magnesium and potassium, both of which are connected to lower blood pressure and maintaining a regular pulse rate. Mangos have a compound known as mangiferin, which has anti-inflammatory properties, they can improve digestion help maintain digestive system. They have both amylase and dietary fiber, which can help relieve constipation. Amylase can break down starches and dissolves other foods in the stomach. Meanwhile, the fiber in mangos can be more effective for relieving constipation than equivalent fiber supplements. Cumin seeds are used in many conditions, including abnormal levels of cholesterol or blood fats dyslipidemia, obesity and many other conditions. Jackfruit seed is taken by mouth as an aphrodisiac or for diabetes. Jackfruit paste is applied to the skin for poisonous bites. Avocado is used for high cholesterol, psoriasis, arthritis, obesity, and many other conditions. Cucumber seed is used for osteoarthritis, reducing high levels of cholesterol, hyperlipidemia, wound healing potentials and hypertension.

\section{UHPLC-HRMS of mango seeds}

Mango seed Irvingia gabonensis mango seeds extract containing ellagic acid, mono, di, tri- $O$ methyl-ellagic acids and their glycosides were analyzed by Ultra-High-Performance liquid chromatography with high-resolution mass spectrometry (UHPLC-HRMS) method [54].

\section{Total phenolic content and antioxidant activities of mango seeds}

Total phenolic content (TPC) antioxidant activities were determined in Mangifera indica belonging to Anacardiaceae family with the use of an HPLC-UV/Vis, gallic acid, caffeic acid, rutin and penta- $O$ galloyl- $\beta$-D-glucose were identified to be present in the mango seed kernel [55].

\section{Antioxidant activity of mango seeds}

Antioxidant activity was determined in Mangifera indica containing gallic acid, polyphenols, flavonoids quercetin, gallic and chlorogenic acids. The extract inhibited the growth of Escherichia coli, Salmonella Typhimurium, Staphylococcus aureus and Listeria monocytogenes. Chromatographic analysis revealed the presence of gallic and chlorogenic acids [56].

Antioxidant, antidiabetic and anticancer properties of cumin seeds

Antioxidant, antidiabetic and anticancer properties were determined in Cumin seeds Cuminum cyminum belonging to Apiaceae family containing p-coumaric acid, ferulic acid, ellagic acid and cinnamic acid [57].

\section{Antihyperlipidemic activity of cumin seeds and Nigella sativa seeds}

Cuminum cyminum powdered Nigella sativa determined the low density lipoprotein cholesterol (LDL) and triglyceride (TG) high density lipoprotein cholesterol (HDL) significantly increased Serum lipids, glucose homeostasis and anthropometric variables in patients with Hashimoto's thyroiditis [58].

\section{A Randomized double-blind placebo-controlled clinical trial of cumin seeds}

A Randomized double-blind placebo-controlled clinical trial was performed on Cuminum cyminum. The weight loss and metabolic profiles among subjects with overweight were studied. Results of the current study indicated that taking high-dose C. cyminum L. plus lime for eight weeks among subjects with overweight had beneficial effects on weight, BMI, FPG, QUICKI, triglycerides, total cholesterol and LDL-cholesterol levels. [59]

\section{Anxiolytic activity of orange seed}

Anxiolytic activity of sweet orange seed Citrus sinensis Rutaceae family containing essential oil aromatherapy for anxiety disorders was determined. Psychologic parameters and physiologic parameters were evaluated before the inhalation period and before, during, and after the Stroop Color-Word Test (SCWT) [60].

\section{Anti-obesity activity of orange seed}

Anti-obesity effects in in vitro and in vivo studies were determined in Citrus sinensis containing anthocyanins, hydroxycinnamic acids, flavone glycosides and ascorbic acid. The study was carried out in overweight healthy human volunteers for $12 \mathrm{w}$. There was a significant reduction in body mass index (BMI), body weight, waist and hip circumference. The results showed that they have a synergistic effect on fat accumulation in humans and the extract can be used in weight management and in the prevention of human obesity [61].

\section{Antidepressant activity of orange seed}

Antidepressant-like effects was studied on Citrus sinensis (L.) containing essential oil Limonene. The results indicated that Orange Essential Oil inhalation significantly ameliorated the depression-like behaviors of chronic unpredictable mild stress (CUMS) mice with decreased bodyweight, sucrose preference, curiosity, and mobility as well as shortened immobile time and attenuated dyslipidemia [62].

\section{Antioxidant and anti-inflammatory activity of orange seed}

Antioxidant and anti-inflammatory in vitro and ex vivo method were determined on Citrus sinensis polyphenolic extract, using the murine macrophages cell line J774A.1 and primary peritoneal macrophages. The in vitro bioavailability of Citrus sinensis polyphenolic extract was assessed using the human cell line Caco-2 grown as monolayers on a transwell membrane [63].

\section{Anti-obesity activity of orange seed}

Anti-Obesity in diet-induced obese zebrafish model was studied on Citrus sinensis containing flavonoid-rich extract of orange juice. In overfed zebrafish model, orange juice significantly decreased both body weight and Body Mass Index values and lowered the visceral adipose tissue, while it had little effect in the normal fed group [64].

\section{Anticancer activity of orange seed and grape seed}

Citrus sinensis and Vitis vinifera polyphenolic extracts in embryonic cardiomyocyte cells (H9c2), and human breast adenocarcinoma cell line (MCF-7) exposed to doxorubicin Possess bioactive polyphenols that protect cardiomyocytes against doxorubicin-induced oxidative stress. The polyphenolic extracts were characterized by Liquid Chromatography-Mass Spectrometry methods [65].

\section{Antidiabetic activity of Jackfruit seed}

Jackfruit seed Artocarpus heterophyllus belonging to Moraceae family, the ethanolic extract $70 \%$ of jackfruit seeds contains betacarotene, epoxide compounds. Jackfruit seeds in reducing blood glucose levels in gestational diabetic rats induced by streptozotocin and in silico study virtual screening through molecular docking were determined [66].

Starch from jackfruit seed powder as superdisintegrant 
Starch from jackfruit seed Artocarpus heterophyllus powder superdisintegrant to design fast dissolving tablets of irbesartan. The tablets prepared from jackfruit seed starch as superdisintegrant were found to be suitable for the preparation of fast dissolving tablets [67].

\section{Antioxidant and antimicrobial activity of Jackfruit seed}

Antioxidant and antimicrobial activity were determined on Artocarpus heterophyllus containing minerals, $\beta$ carotene and dietary fiber, alkaloids, saponins and tannins, phenolic and flavonoid content. GC/MS screening and quantitative analysis of polyphenols, catechin, ascorbic and chlorogenic acids were identified as major polyphenols analyzed by LC-MS/MS and GC/MS analysis [68].

\section{Toxicological and Genotoxic evaluations of avocado seed}

Toxicological and Genotoxic evaluations were determined on ethanolic seed extract of Avocado seed Persea Americana belonging to Lauraceae family [69].

\section{Antioxidant activity of avocado seed}

Antioxidant activity the radical scavenging assay methods ORAC and ABTS were performed on Persea Americana. Methanol and ethanolwater, avocado seed oil, peroxide value and thiobarbituric acid reactive compounds (TBARs) were determined [70].

\section{Liver and kidney functions of avocado seed}

Persea americana Avocado seed powder supplements on meat quality were determined Liver and kidney functions of culled female quails, serum glutamic oxaloacetic transaminase SGOT, and serum glutamic pyruvate transaminase SGPT were also determined. Avocado seed powder supplements improved meat quality as well as the liver and kidney functions of the culled female quail [71].

\section{Antioxidant activity of avocado seed}

Peroxide Value and TBARS (Thiobarbituric acid reactive substances), were analyzed Total polyphenols content (TPC) Oxygen Radical Antioxidant Capacity (ORAC) were determined on Persea Americana [72].

\section{In vitro antioxidant and anticancer effects of avocado seed}

In vitro Antioxidant and Anticancer Effects were determined on Persea americana CASE dose-dependently reduced the viability of human breast (MCF7), lung (H1299), colon (HT29), and prostate (LNCaP) cancer cells [73].

Polyphenolic compounds of Avocado seed were analyzed by UPLC-ESI-MS/MS

Persea Americana containing organic acids, hydroxycinnamic acids, catechins, free and glycosylated flavonoids, and dimeric and trimeric procyanidins. Catechin, epicatechin, six quercetin derivatives, four dimeric procyanidins and three trimeric procyanidins. The polyphenolic-rich extracts and their fractions were analyzed by ultra-performance liquid chromatography-electrospray ionizationmass spectrometry (UPLC-ESI-MS/MS) [74].

\section{In vitro antioxidant and analgesic activities of cucumber seeds}

In vitro antioxidant and analgesic activities were performed on Cucumber seeds Cucumis sativus belonging to Cucurbitaceae family containing flavonoids and tannins. The presence of flavonoids and tannins in the extract might be responsible for free radical scavenging and analgesic effects [75].

\section{Atherosclerosis and hyperlipidemic activity of cucumber seeds}

A Randomized double-blind placebo-controlled clinical trial was studied to determine Atherosclerosis and hyperlipidemic activity on Cucumis sativus. The Total cholesterol, triglycerides (TG), lowdensity lipoprotein (LDL-C), and high-density lipoprotein (HDLC) were evaluated [76].

\section{Antidiabetic activity of Cucumber seeds}

Antidiabetic activity was determined on Cucumis sativus hydroalcoholic and buthanolic extract on Streptozotocin-Induced Diabetic Rats. C. sativus seeds extracts (hydroalcoholic and buthanolic) exhibited excellent antidiabetic activity [77].

\section{CONCLUSION}

This article reviews the functional properties, phytochemical composition of several seeds present in fruits and vegetables. The seeds discussed in this review are compiled from edible fruits, they have the nutritional value and therapeutic efficacy to be used directly as raw material for pharmaceutical formulations and have food value. As some of them contain considerable amounts of alkaloids, glycosides, saponins, flavonoids, terpenoids, tannins, steroids and polyphenolic compounds, as they can become the source for raw materials and lipids. As further investigation is needed in many areas like exploring bioactive phytoconstituents, therapeutic activity, analytical methods, spectroscopic methods and clinical trials, this article can be a future reference for research activities.

\section{RESPONSE TO COMMENTS}

Punctuation errors are rectified cautiously.

\section{AUTHORS CONTRIBUTIONS}

All the authors have equally contributed to the article.

\section{CONFLICT OF INTERESTS}

The authors have no conflict of interest.

\section{FUNDING}

Nil

\section{REFERENCES}

1. Kumoro AC, Alhanif M, Wardhani DH. A critical review on tropical fruits seeds as prospective sources of nutritional and bioactive compounds for functional foods development: a case of indonesian exotic fruits. Int J Food Sci 2020;18:4051475.

2. R Noor Raihana, JMN Marikkar, I Amin, M Shuhaimi. A review on food values of selected tropical fruits' seeds. Int J Food Properties 2015;18:2380-92.

3. Montes Chani EM, Pacheco SOS, Martínez GA, Freitas MR, Ivona $\mathrm{JG}$, Ivona JA, et al. Long-term dietary intake of chia seed is associated with increased bone mineral content and improved hepatic and intestinal morphology in sprague-dawley rats. Nutrients 2018;10:922.

4. Leizer, Cary Ribnicky, David Poulev, Alexander Dushenkov, Vyacheslav, Raskin, et al. The composition of hemp seed oil and its potential as an important source of nutrition. J Nutr Functional Med Foods 2000;2:35-53.

5. Citti C, Linciano P, Panseri S, Vezzalini F, Forni F, Vandelli $\mathrm{MA}$, et al., Cannabinoid profiling of hemp seed oil by liquid chromatography coupled to high-resolution mass spectrometry. Front Plant Sci 2019;10:120.

6. Zhou L, Lin X, Abbasi AM, Zheng B. Phytochemical contents and antioxidant and antiproliferative activities of selected black and white sesame seeds. Biomed Res Int 2016;2016:8495630.

7. Dravie, Emmanuel Elikem, NK Kortei, EK Essuman, CO Tettey, A Boakye, et al. "Antioxidant, phytochemical and physicochemical properties of sesame seed (Sesamum indicum L)." Sci Afr 2020;8:e00349.

8. Mahendra Kumar C, Singh SA. Bioactive lignans from sesame (Sesamum indicum L.): evaluation of their antioxidant and antibacterial effects for food applications. J Food Sci Technol 2015;52:2934-41.

9. Mekky RH, Abdel Sattar E, Segura Carretero A, Contreras MDM. Phenolic compounds from sesame cake and antioxidant activity: new insight for agri-food residues' significance for sustainable development. Foods 2019;8:432.

10. Ruslan K, Happyniar S, Fidrianny I. Antioxidant potential of two varieties of Sesamum indicum L. collected from Indonesia. J Taibah Univ Med Sci 2018;13:211-8. 
11. Ha TJ, Lee MH, Seo WD, Baek IY, Kang JE, Lee JH. Changes occurring in nutritional components (phytochemicals and free amino acid) of raw and sprouted seeds of white and black sesame (Sesamum indicum L.) and screening of their antioxidant activities. Food Sci Biotechnol 2017;26:71-8.

12. Amin MZ, Islam T, Uddin MR, Uddin MJ, Rahman MM, Satter MA. Comparative study on nutrient contents in the different parts of indigenous and hybrid varieties of pumpkin (Cucurbita maxima linn.). Heliyon 2019;5:e02462.

13. Bardaa S, Ben Halima N, Aloui F. Oil from pumpkin (Cucurbita pepo L.) seeds: evaluation of its functional properties on wound healing in rats. Lipids Health Dis 2016;15:73.

14. Abuelgassim AO, Al-showayman SI. The effect of pumpkin (Cucurbita pepo L) seeds and L-arginine supplementation on serum lipid concentrations in atherogenic rats. Afr J Tradit Complement Altern Med 2011;9:131-7.

15. Grzybek M, Kukula Koch W, Strachecka A, Jaworska A, Phiri AM, Paleolog J, et al., K. Evaluation of anthelmintic activity and composition of pumpkin (Cucurbita pepo L.) seed extracts-in vitro and in vivo studies. Int J Mol Sci 2016;17:1456.

16. Chari KY, Polu PR, Shenoy RR. An appraisal of pumpkin seed extract in 1, 2-dimethylhydrazine induced colon cancer in wistar rats. J Toxicol 2018;2018:6086490.

17. Montesano D, Blasi F, Simonetti MS, Santini A, Cossignani L. Chemical and nutritional characterization of seed oil from Cucurbita maxima L. (var. Berrettina) Pumpkin. Foods 2018;7:30.

18. Leverrier A, Daguet D, Calame W, Dhoye P, Kodimule SP. Helianthus annuus seed extract affects weight and body composition of healthy obese adults during $12 \mathrm{w}$ of consumption: a randomized, double-blind, placebo-controlled pilot study. Nutrients 2019;11:1080.

19. Gai F, Karamac M, Janiak MA, Amarowicz R, Peiretti PG. Sunflower (Helianthus annuus L.) plants at various growth stages subjected to extraction-comparison of the antioxidant activity and phenolic profile. Antioxidants (Basel) 2020;9:535.

20. Bhattacharya A, Li Y, Wade KL, Paonessa JD, Fahey JW, Zhang Y. Allyl isothiocyanate-rich mustard seed powder inhibits bladder cancer growth and muscle invasion. Carcinogenesis 2010;31:2105-10.

21. Dua A, Chander S, Agrawal S, Mahajan R. Antioxidants from defatted Indian mustard (Brassica Juncea) protects biomolecules against in vitro oxidation. Physiol Mol Biol Plants 2014;20:539-43.

22. Boscaro V, Boffa L, Binello A. Antiproliferative, proapoptotic, antioxidant and antimicrobial effects of Sinapis nigra L. and Sinapis alba L. extracts. Molecules 2018;23:3004.

23. Mejia Garibay B, Guerrero Beltran JA, Palou E, Lopez Malo A. Physical and antioxidant characteristics of black (Brassica nigra) and yellow mustard (Brassica alba) seeds and their products. Arch Latinoam Nutr 2015;65:128-35.

24. Ahmed AG, Hussein UK, Ahmed AE, Kim KM, Mahmoud HM, Hammouda 0 , et al. Mustard seed (Brassica nigra) extract exhibits antiproliferative effect against human lung cancer cells through differential regulation of apoptosis, cell cycle, migration, and invasion. Molecules 2020;25:2069.

25. Goetz K, Hinz A, Steinhauser J, Von Rath U. Use of mustard seed footbaths for respiratory tract infections: a pilot study. Evid Based Complement Alternat Med 2020;2020:5648560.

26. Farzaneh E, Nia FR, Mehrtash M, Mirmoeini FS, Jalilvand M. The effects of 8-week Nigella sativa supplementation and aerobic training on lipid profile and VO2 max in sedentary overweight females. Int J Prev Med 2014;5:210-6.

27. Albishri HM, Almaghrabi OA, Moussa TA. Characterization and chemical composition of fatty acids content of watermelon and muskmelon cultivars in Saudi Arabia using gas chromatography/mass spectroscopy. Pharmacogn Mag 2013;9:58-66.

28. Mahla HR, Rathore SS, Venkatesan K, Sharma R. Analysis of fatty acid methyl esters and oxidative stability of seed purpose watermelon (Citrullus lanatus) genotypes for edible oil. J Food Sci Technol 2018;55:1552-61.

29. Poduri A, Rateri DL, Saha SK, Saha S, Daugherty A. Citrullus lanatus 'sentinel' (watermelon) extract reduces atherosclerosis in LDL receptor-deficient mice. J Nutr Biochem 2013;24:882-6.
30. Minisy FM, Shawki HH, El Omri A. Pomegranate seeds extract possesses a protective effect against tramadol-induced testicular toxicity in experimental rats. Biomed Res Int 2020;2020:2732958.

31. Aslam MN, Lansky EP, Varani J. Pomegranate as a cosmeceutical source: pomegranate fractions promote proliferation and procollagen synthesis and inhibit matrix metalloproteinase-1 production in human skin cells. J Ethnopharmacol 2006;103:311-8.

32. Torregrosa Garcia A, Avila Gandia V, Luque Rubia AJ, Abellan Ruiz MS, Querol Calderon M, Lopez Roman FJ. Pomegranate extract improves maximal performance of trained cyclists after an exhausting endurance trial: a randomised controlled trial. Nutrients 2019;11:721.

33. Yayla M, Cetin D, Adali Y, Kilicle PA, Toktay E. Potential therapeutic effect of pomegranate seed oil on ovarian ischemia/reperfusion injury in rats. Iran J Basic Med Sci 2018;21:1262-8.

34. Lee HS, Kang P, Kim KY, Seol GH. Foeniculum vulgare mill. Protects against lipopolysaccharide-induced acute lung injury in mice through ERK-dependent NF- $\kappa B$ activation. Korean J Physiol Pharmacol 2015;19:183-9.

35. Bae J, Kim J, Choue R, Lim H. Fennel (Foeniculum vulgare) and Fenugreek (Trigonella foenum-graecum) tea drinking suppresses subjective short-term appetite in overweight women. Clin Nutr Res 2015;4:168-74.

36. Saghafi N, Ghazanfarpour M, Khadivzadeh T, Babakhanian M, Afiat M. The effect of Foeniculum Vulgare (Fennel) on body composition in postmenopausal women with excess weight: a double-blind randomized placebo-controlled trial. J Menopausal Med 2017;23:166-71.

37. Mohamad RH, El-Bastawesy AM, Abdel-Monem MG, Noor AM, AlMehdar HA, Sharawy SM, et al. Antioxidant and anticarcinogenic effects of methanolic extract and volatile oil of fennel seeds (Foeniculum vulgare). J Med Food 2011;14:986-1001.

38. Sadrefozalayi S, Farokhi F. Effect of the aqueous extract of Foeniculum vulgare (fennel) on the kidney in experimental PCOS female rats. Avicenna J Phytomed 2014;4:110-7.

39. Bruce Keller AJ, Richard AJ, Fernandez Kim SO. Fenugreek counters the effects of high-fat diet on gut microbiota in mice: links to metabolic benefit. Sci Rep 2020;10:1245.

40. Ranade M, Mudgalkar N. A simple dietary addition of fenugreek seed leads to the reduction in blood glucose levels: a parallelgroup, randomized single-blind trial. Ayu 2017;38:24-7.

41. Pundarikakshudu K, Shah DH, Panchal AH, Bhavsar GC. Antiinflammatory activity of fenugreek (Trigonella foenumgraecum linn) seeds petroleum ether extract. Indian J Pharmacol 2016;48:441-4.

42. Prommaban A, Utama-Ang N, Chaikitwattana A, Uthaipibull C, Porter JB, Srichairatanakool S. Phytosterol, lipid and phenolic composition, and biological activities of guava seed oil. Molecules 2020;25:2474.

43. Panzarini E, Dwikat M, Mariano S, Vergallo C, Dini L. Administration dependent antioxidant effect of Carica papaya seeds water extract. Evid Based Complement Alternat Med 2014;2014:281508.

44. Okeniyi JA, Ogunlesi TA, Oyelami OA, Adeyemi LA. Effectiveness of dried Carica papaya seeds against human intestinal parasitosis: a pilot study. J Med Food 2007;10:194-6.

45. Zhou K, Wang H, Mei W, Li X, Luo Y, Dai H. Antioxidant activity of papaya seed extracts. Molecules 2011;16:6179-92.

46. Yismaw G, Tessema B, Mulu A, Tiruneh M. The invitro assessment of the antibacterial effect of papaya seed extract against bacterial pathogens isolated from urine, wound and stool. Ethiop Med J 2008;46:71-7.

47. Zeb A. Phenolic profile and antioxidant activity of melon (Cucumis Melo L.) seeds from Pakistan. Foods 2016;5:67.

48. Ganji SM, Singh H, Friedman M. Phenolic content and antioxidant activity of extracts of 12 melon (Cucumis melo) peel powders prepared from commercial melons. J Food Sci 2019;84:1943-8.

49. Vella FM, Cautela D, Laratta B. Characterization of polyphenolic compounds in cantaloupe melon by-products. Foods 2019;8:196. 
50. Zhang X, Bai Y, Wang Y, Wang C, Fu J, Gao L, et al. Anticancer properties of different solvent extracts of Cucumis melo L. seeds and whole fruit and their metabolite profiling using HPLC and GC-MS. Biomed Res Int 2020;2020:5282949.

51. John OD, Mouatt P, Majzoub ME, Thomas T, Panchal SK, Brown L. Physiological and metabolic effects of yellow mangosteen (Garcinia dulcis) rind in rats with diet-induced metabolic syndrome. Int J Mol Sci 2019;21:272.

52. Xie Z, Sintara M, Chang T, Ou B. Daily consumption of a mangosteen-based drink improves in vivo antioxidant and antiinflammatory biomarkers in healthy adults: a randomized, double-blind, placebo-controlled clinical trial. Food Sci Nutr 2015;3:342-8.

53. Hiranrangsee L, Kumaree KK, Sadiq MB, Anal AK. Extraction of anthocyanins from pericarp and lipids from seeds of mangosteen (Garcinia mangostana L.) by ultrasound-assisted extraction (UAE) and evaluation of pericarp extract enriched functional ice-cream. J Food Sci Technol 2016;53:3806-13.

54. Sun J, Chen P. Ultra high-performance liquid chromatography with high-resolution mass spectrometry analysis of African mango (Irvingia gabonensis) seeds, extract, and related dietary supplements. J Agric Food Chem 2012;60:8703-9.

55. Lim KJA, Cabajar AA, Lobarbio CFY, Taboada EB, Lacks DJ. Extraction of bioactive compounds from mango (Mangifera indica L. var. Carabao) seed kernel with ethanol-water binary solvent systems. J Food Sci Technol 2019;56:2536-44.

56. Bernal Mercado AT, Ayala Zavala JF, Cruz Valenzuela MR. Using sensory evaluation to determine the highest acceptable concentration of mango seed extract as antibacterial and antioxidant agent in fresh-cut mango. Foods 2018;7:120.

57. Arun KB, Aswathi U, Venugopal VV, Madhavankutty TS, Nisha P. Nutraceutical properties of cumin residue generated from Ayurvedic industries using cell line models. J Food Sci Technol 2016;53:3814-24.

58. Farhangi MA, Dehghan P, Tajmiri S. Powdered black cumin seeds strongly improves serum lipids, atherogenic index of plasma and modulates anthropometric features in patients with Hashimoto's thyroiditis. Lipids Health Dis 2018;17:59.

59. Taghizadeh M, Memarzadeh MR, Abedi F. The effect of Cumin cyminum L. Plus lime administration on weight loss and metabolic status in overweight subjects: a randomized double-blind placebocontrolled clinical trial. Iran Red Crescent Med J 2016;18:e34212.

60. Goes TC, Antunes FD, Alves PB, Teixeira Silva F. Effect of sweet orange aroma on experimental anxiety in humans. J Altern Complement Med 2012;18:798-804.

61. Cardile V, Graziano AC, Venditti A. Clinical evaluation of moro (Citrus sinensis (L.) Osbeck) orange juice supplementation for the weight management. Nat Prod Res 2015;29:2256-60.

62. Zhang LL, Yang ZY, Fan G, Ren JN, Yin KJ, Pan SY. Antidepressant-like effect of citrus sinensis (L.) osbeck essential oil and its main component limonene on mice. J Agric Food Chem 2019;67:13817-28.

63. Pepe G, Pagano F, Adesso S, Sommella E, Ostacolo C, Manfra M, et al. Bioavailable Citrus sinensis extract: polyphenolic composition and biological activity. Molecules 2017;22:623.
64. Montalbano G, Mania M, Guerrera MC, Laura R, Abbate F, Levanti M, et al. Effects of a flavonoid-rich extract from Citrus sinensis juice on a diet-induced obese zebrafish. Int J Mol Sci 2019;20:5116.

65. Pepe G, Salviati E, Rapa SF, Ostacolo C, Cascioferro S, Manfra M, et al. Citrus sinensis and vitis vinifera protect cardiomyocytes from doxorubicin-induced oxidative stress: evaluation of onconutraceutical potential of vegetable smoothies. Antioxidants (Basel) 2020;9:378.

66. Dwitiyanti D, Rachmania RA, Efendi K, Septiani R, Jihadudin P. In vivo activities and in silico study of jackfruit seeds (Artocarpus heterophyllus lam.) on the reduction of blood sugar levels of gestational diabetes rate induced by streptozotocin. Open Access Maced J Med Sci 2019;7:3819-26.

67. Suryadevara V, Lankapalli SR, Danda LH, Pendyala V, Katta V. Studies on jackfruit seed starch as a novel natural superdisintegrant for the design and evaluation of irbesartan fast-dissolving tablets. Integr Med Res 2017;6:280-91.

68. Sharma A, Gupta P, Verma AK. Preliminary nutritional and biological potential of Artocarpus heterophyllus L. shell powder. J Food Sci Technol 2015;52:1339-49.

69. Padilla Camberos E, Martinez Velazquez M, Flores Fernandez JM, Villanueva Rodriguez S. Acute toxicity and genotoxic activity of avocado seed extract (Persea americana Mill., c. v. Hass). Sci World J 2013;2013:245828.

70. Segovia FJ, Hidalgo GI, Villasante J, Ramis X, Almajano MP. Avocado seed: a comparative study of antioxidant content and capacity in protecting oil models from oxidation. Molecules 2018;23:2421.

71. Tugiyanti E, Iriyanti N, Apriyanto YS. The effect of avocado seed powder (Persea americana Mill.) on the liver and kidney functions and meat quality of culled female quail (Coturnix coturnix japonica). Vet World 2019;12:1608-15.

72. Gomez FS, Sanchez SP, Iradi MG, Azman NA, Almajano MP. Avocado seeds: extraction optimization and possible use as antioxidant in food. Antioxidants (Basel) 2014;3:439-54.

73. Dabas D, Elias RJ, Ziegler GR, Lambert JD. In vitro antioxidant and cancer inhibitory activity of a colored avocado seed extract. Int J Food Sci 2019;2019:6509421.

74. Rosero JC, Cruz S, Osorio C, Hurtado N. Analysis of phenolic composition of byproducts (seeds and peels) of avocado (Persea americana Mill.) cultivated in colombia. Molecules 2019;24:3209.

75. Kumar D, Kumar S, Singh J. Free radical scavenging and analgesic activities of Cucumis sativus L. fruit extract. J Young Pharm 2010;2:365-8.

76. Soltani R, Hashemi M, Farazmand A, Asghari G, Heshmat Ghahdarijani K, Kharazmkia A, et al. Evaluation of the effects of Cucumis sativus seed extract on serum lipids in adult hyperlipidemic patients: a randomized double-blind placebocontrolled clinical trial. J Food Sci 2017;82:214-8.

77. Minaiyan M, Zolfaghari B, Kamal A. Effect of hydroalcoholic and buthanolic extract of Cucumis sativus seeds on blood glucose level of normal and streptozotocin-induced diabetic rats. Iran J Basic Med Sci 2011;14:436-42. 